\title{
ORIENTATION VS. ORIENTATION: IMAGE PROCESSING FOR STUDIES OF DENTAL MORPHOLOGY
}

\author{
A.V. Gaboutchian ${ }^{1 *}$, V. A. Knyaz ${ }^{2,3}$, S.V. Vasilyev ${ }^{4}$, D.V. Korost ${ }^{5}$, A.A. Kudaev ${ }^{5}$ \\ ${ }^{1}$ Peoples' Friendship University of Russia, 117198, Moscow, Russia - armengaboutchian@ mail.ru \\ ${ }^{2}$ State Research Institute of Aviation System (GosNIIAS), 125319 Moscow, Russia - knyaz@gosniias.ru \\ ${ }^{3}$ Moscow Institute of Physics and Technology (MIPT), Dolgoprudny, Russia \\ ${ }^{4}$ Institute of Ethnology and Anthropology RAS, Moscow, Russia - vasbor1@ yandex.ru \\ ${ }^{5}$ Faculty of Geology, Moscow State University, 119234, Moscow, Russia - dkorost@mail.ru; a.a.kudaev@gmail.com
}

\section{Commission II, WG II/10}

KEY WORDS: automated digital odontometry (aDo), micro-CT, 3d reconstructions of teeth, palaeoanthropology, Sunghir, object orientation

\begin{abstract}
:
Many odontological studies held through application of traditional and modern techniques, especially when related to measurements and morphology, very much depend on methodological aspects referred to orientation of teeth. And this is particularly relevant to new imaging and $3 \mathrm{~d}$ reconstruction implemented in dental research and practice in a wide range of disciplines from anthropology to dentistry. The current paper deals with studies of palaeoanthropological findings dating back to the Upper Palaeolithic period in Central Russia - well-known archaeological site of Sunghir. Micro-computed tomography has been used for digital reconstructions of teeth - molars and premolars representing well-preserved dental morphology of an adolescent individual. This is due to new opportunities introduced by $3 \mathrm{~d}$ reconstruction techniques in general and high-resolution $\mathrm{x}$-ray imaging in particular that this study has become relevant. Thus digital techniques do not only provide for operating convenience but, which is even more important, allow application of image processing algorithms. In the suggested methodology these are automated, based on morphological interpretations and serve for orientation of studied teeth for further measurements. At the same time micro-computed tomographic imaging allows accurate reconstruction of other morphologically important structures which are used for an alternative orientation algorithm. Comparisons of dental measurements' results obtained through automated digital odontometry $(\mathrm{aDo})$ after both orientations applied are presented in the current paper.
\end{abstract}

\section{INTRODUCTION}

Traditionally morphological descriptions have been based on visual characterization. The most essential features are classified in accordance with their expression degree. A number of such features corresponding to teeth of various groups form the morphological portrait of the studied tooth. However more profound, precise and objective research usually requires wider range of approaches involving technological cooperation, imaging and mathematical processing. Among such we can mention different measurement techniques and morphometric analysis. These study techniques are widely used in various spheres of biological research including studies of dental morphology for palaeoanthropological applications (Bae et al., 2014; Kaifu et al., 2015; Hershkovitz et al., 2018). And it should be noted that both of the above-mentioned methods have received a strong impetus with the development of imaging techniques and image processing allowing introduction of $3 \mathrm{~d}$ reconstructions and their analysis. Despite current availability of multiple approaches to imaging (variety of optical techniques, different lighting and processing methods) the leading role for morphological studies should be given to $\mathrm{x}$-ray based micro-computed tomography. And the reasons for this lies in the combination of such advantages as potential in achieving high accuracy of reconstructions, full coverage of morphologically and histologically important layers of tissues (including outer and inner surfaces), as well as in providing for

\footnotetext{
${ }^{*}$ Corresponding author
}

non-destructive imaging techniques and non-invasive nature of research. Nevertheless optical imaging has been successfully implemented in a number of previously conducted studies providing detailed outer surface reconstructions which were sufficient for development of bases for methods discussed in the current article.

The mentioned above varieties of research methods (distance measurement or shape analysis) and image obtaining principles for $3 \mathrm{~d}$ reconstruction (optical or $\mathrm{x}$-ray) have led to state of affairs when data collection which is always in close linkage with the methodological approaches applied to spatial orientation of the studied objects. The reason is that the object orientation impacts all further procedures such as measurer positioning, landmark setting, contour demarcating, sectioning and others. Such factors as improvements in imaging techniques, increase of $3 \mathrm{~d}$ reconstruction accuracy, application of digital image processing and analysis inevitably empower the research potential on one hand and increase requirements toward methodological standards. Thus if we refer to the article topic, accurate orientation becomes even more important, and changes in the obtained study results can be presumably tracked and associated. In a number of our previous studies orientation has been performed automatically, it has been based on original interpretation of basic dental morphology and $3 \mathrm{~d}$ surface analysis. And currently we apply automated algorithms to the enamel cervical margin (ECM) and compare its performance with conventional, based on occlusal surface contour 
(OSC) orientation. These approaches to orientation are referred to the method of automated digital odontometry and serve for subsequent launching of sectioning and measuring algorithms. Measurements results obtained in both cases of orientation are compared.

\section{RELATED WORKS}

Orientation procedures for dental measurements are usually performed on the basis of traditionally accepted methodologies which serve for assessment of two main parameters: bucco-lingual and mesio-distal crown diameters. Methodological recommendations include guidelines for positioning of measuring instrument (odontometer) in accordance with the measured tooth type and its handled position. This allows correct setting of points limiting the measured distance (Zubov, 1968 and 2006; Hillson, 2003). Similar techniques can be applied to $3 \mathrm{~d}$ reconstructions of teeth duplicating measurements performed on real objects such as teeth or plaster copies of dental arches (Zilberman et al., 2003, Naidu and Freer, 2013; Zhang et al., 2016). Digital techniques in their turn allow "manual" and automated modes of orientation and measurements. And these methods have gone far beyond the limits of traditional odontometry providing sufficient amount and variety of parameters allowing morphological description of teeth (Gaboutchian et al., 2020).

Another direction of odontological research dealing with orientation of teeth is related to the application of geometric morphometric analysis. And in a number of such dental morphological studies the initial stage of tooth analysis is referred to detection of enamel cervical margin (ECM) and construction of cervical plane which the best-fit for the line representing the above-mentioned margin. Despite the fact the plane is horizontal it serves for setting vertical orientation of the studied tooth and further constructions are performed around vertical z-axis perpendicular to that plane (Benazzi et al., 2012; Krenn et al., 2019). In addition variations in setting the cervical plane have been described methodologically. Thus as an example there is an approach based on setting ECM as well and positioning the cervical plane on the most apical part on the cervical line contour for obtaining a continuous ring of enamel. Moreover multiple combinations of approaches for calculating the cervical plane inclination have been suggested as well.

The above-mentioned methodological variations become even more diverse especially with regard to studies of dental enamel thickness (Suwa and Kono, 2005; Schwartz and Dean, 2005). Availability of some morphological structures is limited and depends to some extent on imaging technique. Thus an alternative horizontal plane positioning and orientation implies it being rested on main dental horn tips (Tafforeau (2004) in Benazzi et al., 2014) which can't be directly reconstructed neither through contact scanning nor optical imaging but through $\mathrm{x}$-ray based scanning only. This method of orientation is able to considerably impact the measurement results as inclination of such plane can be significantly different from all types of approaches using enamel cervical margin for orientation. After horizontal plane settings subsequent stages refer to orientation of vertical plane (or planes). The latter serve for sectioning teeth and further enamel thickness measurements on $2 \mathrm{~d}$ contours. Contour obtaining requires orientation of vertical plane (it is usually set perpendicular to horizontal) according to morphological structures typically referred to cusp tips or their pairs especially when applied to traditional physical sectioning of teeth (Martin, 1983). 3d reconstructions obtained through high-resolution x-ray imaging allow accurate setting of vertical planes through dentin horn tips (Feeney et al., 2010). Such techniques have been developed and suggested for teeth classified into groups according to their positions in dental arches, and one or two planes are set depending on the tooth type (Benazzi et al., 2014).

High resolution $\mathrm{x}$-ray imaging and $3 \mathrm{~d}$ reconstruction techniques considerably expand opportunities of odontological studies. And this refers to orientation protocols as well, as they can be now based on previously unavailable morphological structures or structures more accurately and clearly detected after application of appropriate imaging and reconstruction techniques. In addition, volume and surface area assessments technically feasible on $3 \mathrm{~d}$ reconstructions become essential parts of studies of enamel thickness research avoiding application of invasive and destructive techniques (Olejniczak, 2008; Suwa et al., 2009). Significantly more detailed research becomes possible in odontology in general and including geometric morphometric analysis as well (Ortiz et al., 2019).

Enamel cervical margin (ECM) is often used as a setting directions in odontology, nevertheless it is not the only reliable morphological structure for orientation launching. Starting from the above-mentioned manual measurement techniques to measurement based on micro-CT tomographic scanning alignment of teeth according to their vertical axis and occlusal view outline has been used. Thus with regard to permanent molars the reference or orientational plane can be identified by iterative oscillation of the model to view the maximum occlusal surface (Kono, 2004; Suwa and Kono, 2005). This approach has been debated and cautioned about, because not entirely independent of the enamel thickness to be measured (see Olejniczak (2006) in Benazzi et al., 2014). However it should be noted that the occlusal surface possesses high morphologically important potential providing sufficient data not only for orientation but even for development of automated orientational algorithms based on $3 \mathrm{~d}$ surface analysis. These methods are based on outlining the contour of anatomical occlusal surface after staged surface curvature analysis. These automated methods serve for detailed morphological studies performed through measurements (Gaboutchian et al., 2020) which can be performed both enamel and dentin surfaces (Gaboutchian et al, 2021). The basis of the mentioned techniques were developed and tested on $3 \mathrm{~d}$ reconstructions of teeth and dental obtained through optical imaging techniques. Nevertheless the suggested methodology allowed to use reconstructions obtained through micro-CT, including inner morphological structures.

The described above orientation protocols refer to positioning teeth or their $3 \mathrm{~d}$ reconstructions which can be subsequently measured or sectioned by correctly (according to the methodology) positioned measurers or sectioning planes. Nevertheless geometric constructions and measurements on the contours obtained are a matter of discussion as well. Orientation is a necessary stage for $2 \mathrm{~d}$ assessments as well, and these algorithms are described as automated for measurements of teeth (Gaboutchian et al., 2019) or serving for enamel thickness measurements (Suwa and Kono, 2005; Smith et al., 2018).

\section{MATERIALS AND METHODS}

The study has been conducted on $3 \mathrm{~d}$ reconstructions of teeth from the Upper Palaeolithic burial of Sunghir. The site has been discovered in the late fifties of the last century. It is named after a 
tributary to the Klyazma River that flows in the Central regions of Russia. One of two adolescents discovered in a paired burial that contained a large number of artefacts and excellently preserved remains. Teeth of the elder individual Sunghir-2 served for studies in the current research. The dental arches contained almost a complete set of permanent teeth with preserved, due to the individuals' young age, morphology.

For obtaining digital reconstructions of teeth the skull of the individual was placed in Phoenix $v \mid$ tome $\mid x$ (General Electric) tomographic scanner and subjected to x-ray imaging. Stacks of parallel slices were generating in .bmp format, 8-bit colour depth and with $43 \mu \mathrm{m}$ inter-slice distance. For reconstructing separate teeth images related to each tooth were extracted from the entire stack and subsequently processed independently. $3 \mathrm{~d}$ reconstruction was performed by means of Avizo 9.01 software (Thermo Fisher Scientific). Segmentation procedures based on gradients of x-ray absorption for different tissues (enamel possesses higher level of absorption in comparison with dentin) were performed by application of "Threshold tool". Afterwards morphologically irrelevant areas, though possessing the same absorption level, were separated from dental tissue reconstructions and cleaned. Subsequently $3 \mathrm{~d}$ reconstructions were generated within the allocated parts of $2 \mathrm{~d}$ image stack; the process was accompanied by surface smoothing procedures. Reduction of each model size was performed at the surface simplification step; tetrahedrons were generated from voxel though "Simplification editor". Lastly the models were converted to .stl and .x formats for further processing: orientation and measurements. $3 \mathrm{~d}$ reconstructions of two Sunghirian teeth, or rather their enamel caps, were measured for this study. These are the upper right first premolar (tooth c2_14) and upper right third molar (tooth c2_18). The latter - wisdom tooth - possesses intact surface as it hasn't been erupted due to individual's age.

This research was performed by means of different versions of software (Knyaz, 2012, Knyaz, 2016) developed in the State Research Institute of Aviation System (GosNIIAS). This application allowed uploading $3 \mathrm{~d}$ reconstructions of teeth, their surface analysis, different modes of sectioning, contour measurements as well as data saving and analysis; these functions are automated. Corrections in the manual mode can be performed as well. The current study algorithm includes loading of tooth enamel cap 3d reconstruction, its orientation according to anatomical occlusal surface contour, sectioning according to the orientation set for subsequent contour obtaining and measurements on these contours. These procedures are fully automated. After saving the measurement results the above-mentioned algorithm has been repeated with respect to orientation performed according to enamel cervical margin contour. The results of measurement obtained after both approaches to orientation were compared. Orientation algorithms are based on surface curvature analysis, which allows finding areas where centrally located occlusal surface bends towards the outer walls of the tooth crown delineating the contour of anatomical occlusal surface (OSC). Enamel cervical margin is set in a similar manner as the most remote points of the reconstruction located at the junction of inner and outer surfaces of tooth enamel cap (Figure $1 \mathrm{a}, \mathrm{b}$ ).

Further stages of surface analysis through automated algorithms provide orientation of teeth on the basis of their occlusal surface contour (OSC) and enamel cervical margin (ECM). These structures provide data for vertical axis orientation, which is indicated in green colour at the presented figures (Figure $2 \mathrm{a}, \mathrm{b}$ ). Other axes (yellow-blue and red) are oriented in accordance with the

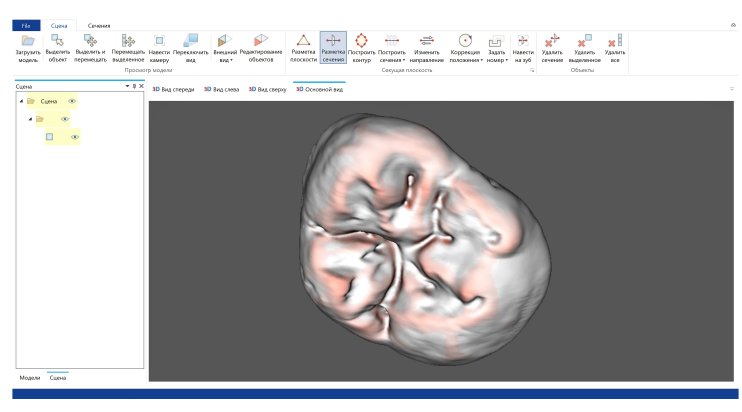

(a) top view

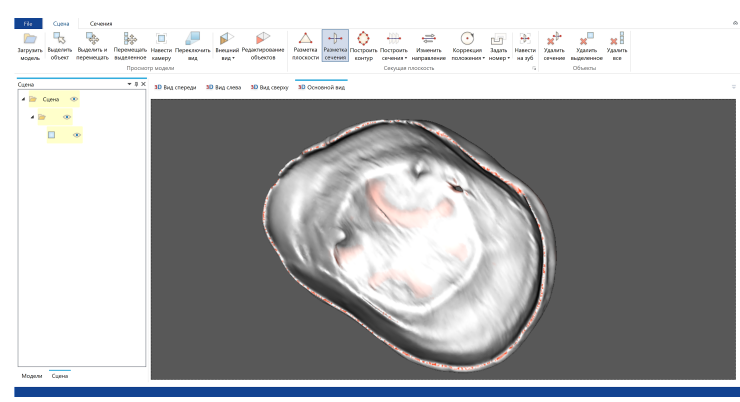

(b) bottom view

Figure 1. Enamel cap of upper right third molar, C2 Suhghir; top view (a) and bottom view (b)

occlusal surface contour shape; in some cases, taking into consideration variety of types of teeth, mesio-distal axis direction (yellow-blue) requires manual adjustments.

The currently studied teeth as discrete objects didn't require any manual adjustments regarding mesio-distal axis as it was set automatically in a accordance with the suggested methodology. Nevertheless some manual correction mesio-distal axis was performed in order to minimise discrepancies in measurements caused by its shift which inevitably accompanies displacement of vertical axis while re-orientating the studied teeth vertically. Thus the maximal emphasis was on two vertical orientation's difference ECM and OSC versions which resulted in defining contours of the above-mentioned morphological structures and setting starting conditions for sectioning teeth (Figure $3 \mathrm{a}, \mathrm{b}$ ).

All further procedures were performed according to algorithms of automated digital odontometry (aDo). Parallel sections with equal intervals are set perpendicular to mesio-distal axis (yellowblue on the figures). This procedure creates of contours of the studied enamel caps which in their turn serve for measuring variety of parameters (Figure 4). 50-section mode was applied; 3 marginal sections from both mesial and distal sides were excluded from the measurements not to interfere measurement results. Only average parameters, obtained as arithmetic mean of the same parameters on all sections, were analysed.

\section{RESULTS AND DISCUSSION}

Application of high resolution x-ray imaging can significantly improve palaeoanthropological research providing wide and accurate access to morphology. Hence with respect to the currently studied $3 \mathrm{~d}$ reconstructions, they contain all necessary data for delineating the enamel cervical margin (ECM). In cases of use of other imaging techniques clear detection of enamel cervical margins has its limitations impacting studied sample selection. Thus 


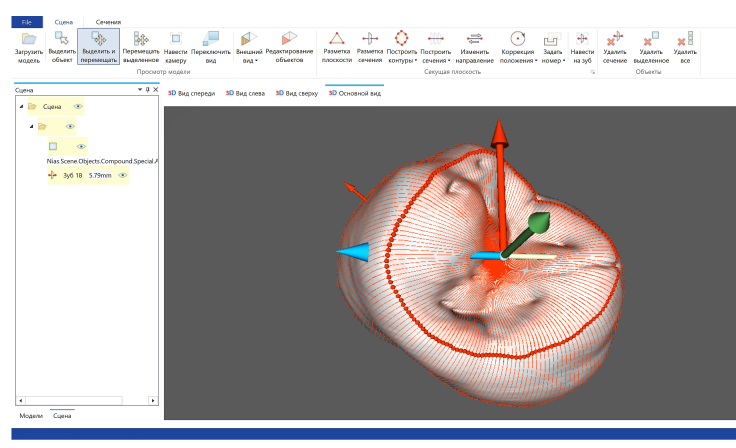

(a) occlusal surface contour

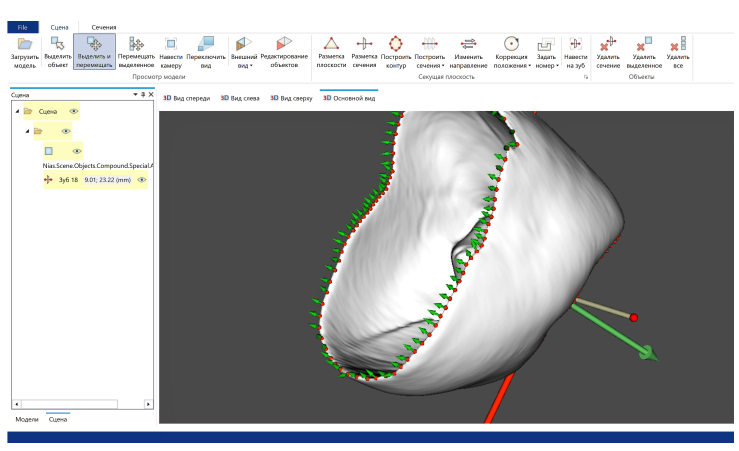

(b) enamel cervical margin

Figure 2. Intermediate results of orientation according to occlusal surface contour (OSC) set on upper right first premolar of Suhghir-2 (a) and enamel cervical margin (ECM) on the same tooth.

reconstructions obtained through intra-oral or optical scanning of stone cast models of teeth and dental arches do not convey the enamel edge contour either due to imaging technique potential or for the reason that the ECM might be partially or completely covered by soft tissues. As another factor we should mention the curved contour of the ECM which makes problematic application of this morphological structure for orientation in the process of manual measurements. In general, dental measurements contain variety of difficulties and alternative approaches caused not only by features of imaging techniques but primarily by the dental morphology. The point is that teeth are of different types, conditions, degree of wear; they might belong to different species, found in different regions and, in addition, possess natural biological variability in between individuals. They are difficult objects to measure and it is important for measuring technique to reflect the principles of dental morphology.

Taking into consideration a lack of comprehensive odontontometric methodology covering at least a significant part of the dental morphological variety existing of alternative measurement techniques is unavoidable and, to some extent, necessary. And they are certainly based on different morphological approaches developing in line with general direction of technological advances, such as new imaging techniques. Application of alternative morphological bases for orientation and measurements can provide for accuracy, coverage of wider samples in terms of their amount as well as $3 \mathrm{~d}$ reconstruction method, variety of study aims etc. Improvements referring orientation applied to micro-CT $3 \mathrm{~d}$ reconstructions have brought to development of new algorithms based on the enamel cervical margin (ECM) analysis which can be compared to traditionally (this refers to the method of automated digital odontometry - aDo) applied orientation in accor-

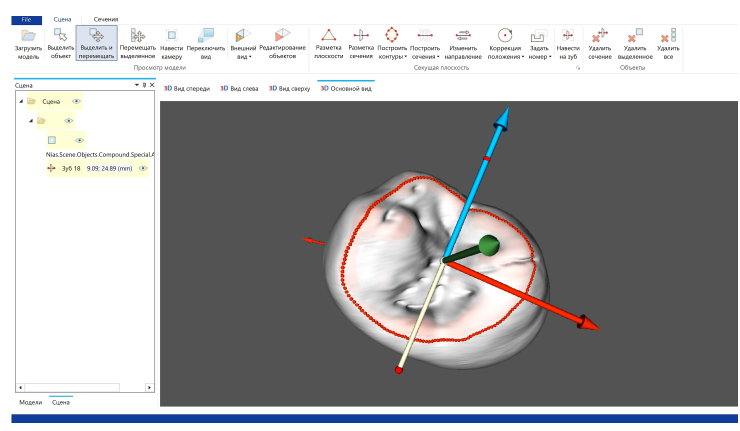

(a) occlusal surface contour

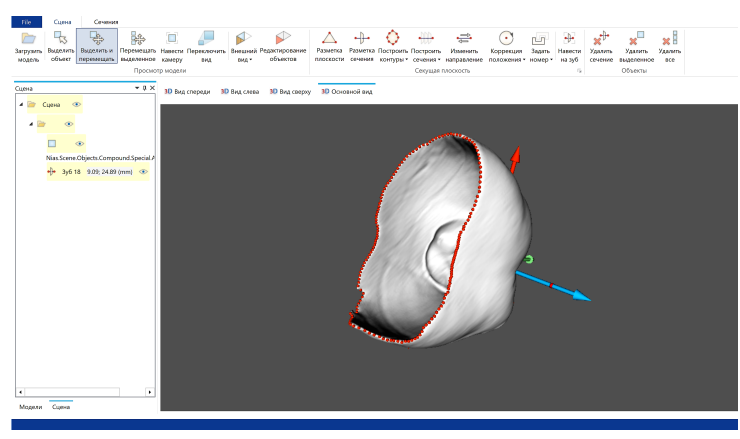

(b) enamel cervical margin

Figure 3. Final results of orientation according to occlusal surface contour (OSC) set on upper right first premolar of Suhghir-2 (a) and enamel cervical margin (ECM) on the same tooth.

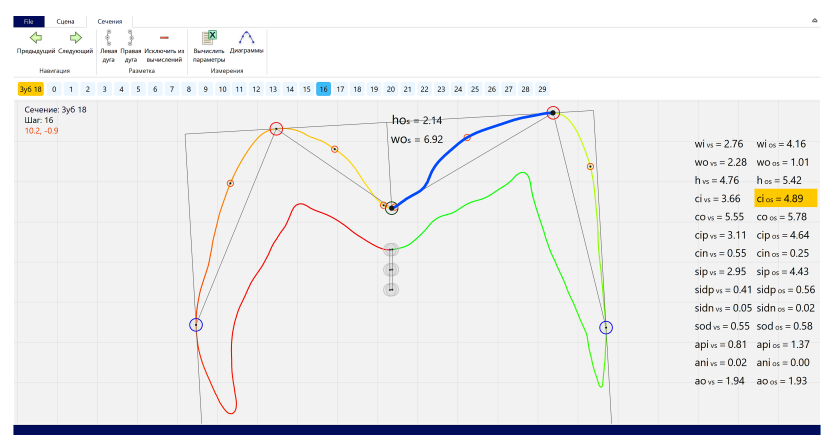

Figure 4. Measurements on the enamel cap contour.

dance with occlusal surface contour (OSC).

Each of the two orientation launching structures possesses advantages and disadvantages. Thus OSC is the structure which can be reliably detected on various teeth: premolars and molars, upper and lower, worn out or intact. However the occlusal surface is always is in the process of functioning and gradual changes up to severe dental wear. Can it be perceived as a positive feature for orientation or no is a matter of discussion. On one hand such orientation reflects the real condition of the studied tooth and changes in line with morphological conditions though it might seem instable on the other. It is very important to emphasize significant integrative meaning of anatomical occlusal surface which allows to unite different parts of teeth in terms of both morphology and function. This is an important aspect for preserving linkage between the study method and the studied object. Moreover, anatomical occlusal surface can be clearly observed and reconstructed regardless of the imaging technique. Nevertheless with the help of the above-mentioned imaging techniques 
another morphological structure can be clearly defined - enamel cervical margin (ECM) which is widely used for orientation of teeth in palaeoanthropological research. We can assume that the ECM possesses higher stability if compared with the occlusal surface undergoing obvious changes. However there is a lack of data related to changes of the ECM during the lifetime which actually might happen as non-caries lesions of teeth are localised around the cervical region. Regardless of tooth type and other conditions the ECM is rather curved, hence on premolars, canines and incisors its degree might be a hindering factor for horizontal plane construction. Apparent stability of ECM is combined in the majority of techniques with manual adjustments for orientation which requires subsequent vertical plane constructions. The latter usually require a high degree of morphological integrity of occlusal surface as this stage of orientation is performed according to cusp or dentin horn tips' position. This fact is not only emphasises the morphological importance of occlusal surface but significantly limits the potentially studied sample to finely preserved only. Cusp tips are affected by dental wear and can be completely eliminated in the result of the process. In more severe dental wear cases dentin horn tips are involved as well. Of course, occlusal surface is the main area of changes caused by chewing and other functional and non-functional acts of masticatory system. Nevertheless the morphological pattern of anatomical occlusal surface remains the same - it is the inner, deeper and centrally located part dental crown possessing borders beyond which outer surfaces of the crown bend towards roots. This has determined that the leading role in orientation of teeth in the presented method of automated digital odontometry belongs to the OSC. Nevertheless the suggested algorithms are able to consider in the orientation process an additional structure of ECM which has become clearly defined due to the applied imaging technique.

The measurement result presented below in Table 1 are obtained on two described above teeth.

\begin{tabular}{lcccc}
\hline \hline & $1.4 / \mathrm{OSC}$ & $1.4 / \mathrm{ECM}$ & $1.8 / \mathrm{OSC}$ & $1.8 / \mathrm{ECM}$ \\
\hline \multicolumn{5}{c}{ linear horizontal, mm } \\
\hline tooth & 10.043 & 10.019 & 12.418 & $\mathbf{1 2 . 4 2 0}$ \\
oral cusps & 5.725 & $\mathbf{5 . 7 3 9}$ & 6.627 & 6.620 \\
vest. cusps & 4.299 & 4.280 & 5.791 & $\mathbf{5 . 8 0 0}$ \\
occl. surface & 5.714 & 5.573 & 7.221 & 7.210 \\
\hline \multicolumn{5}{c}{ linear vertical, mm } \\
\hline occl. surface & 1.81 & 1.779 & 2.102 & $\mathbf{2 . 1 0 4}$ \\
\hline \hline
\end{tabular}

Table 1. Linear measurements for teeth oriented in the two modes (OSC and ECM)

Vertical axis inclination has been performed in two modes. First OSC is calculated on the basis of occlusal surface contour which has been subsequently corrected according to enamel cervical margin (ECM mode). The procedures were conducted through automated algorithms initially permitting manual adjustments, however at the current stage of software development completely automated. Re-orientation of teeth impacts the obtained results which are presented only as average linear parameters. In 6 out 10 presented parameters cause decrease of the absolute figures (italic font). This decreasing pattern is more characteristic for the premolar; hence the molar measurements perceive changes to orientation mode as increasing the sizes of the measured structures (bold font). The only coinciding parameter behaviour pattern on both teeth refers to horizontal dimension of anatomical occlusal surface as it decreases on premolar (from 5.714 to 5.573) and on molar (from 7.221 to 7.210 ). It might be noted that the changes are insignificant; however the measurement method sensitivity allows to register the described differences.

Larger sample of teeth is required for similar studies as the two tested orientation modes can demonstrate different behaviour on teeth with distinct morphological changes caused by functional or non-functional dental wear.

\section{CONCLUSION}

Involving of high resolution x-ray based imaging techniques in palaeoanthropological studies of teeth provides for more complete reconstruction of dental morphology. This can be a useful feature for dental research especially when combined with new method of measurements - automated digital odontometry which is empowered by image processing provided by the applied software. A wider coverage of morphological structures can be performed at the orientation stage of measurements, which impacts the obtained results. A matter of orientation mode choice should be studied in further more profound studies including wider sample of teeth.

\section{REFERENCES}

Bae et al., 2014. Bae, Ch., Wang, W., Zhao, J-X., Huang, Sh., Tian, F., Shen, G. Modern human teeth from Late Pleistocene Luna Cave (Guangxi, China). Quaternary International. 354. 10.1016/j.quaint.2014.06.051

Benazzi et al., 2012. Benazzi, S., Fornai, C., Buti, L., Toussaint, M., Mallegni, F., Ricci, S., Gruppioni, G., Weber, G., Condemi, S., Ronchitelli, A. (2012). Cervical and Crown Outline Analysis of Worn Neanderthal and Modern Human Lower Second Deciduous Molars. American journal of physical anthropology. 149. 10.1002/ajpa.22155

Benazzi et al., 2014. Benazzi, S., D. Panetta, C. Fornai, M. Toussaint, G. Gruppioni, and J. Hublin (2014). Technical Note: Guidelines for the Digital Computation of 2D and 3D Enamel Thickness in Hominoid Teeth. American Journal of Physical Anthropology, 153: 305-313

Feeney et al., 2010. Feeney R.N.M., Zermeno J.P., Reid D.J., Nakashima S., Sano H., Bahar A., Hublin J.-J., Smith T.M.. Enamel thickness in Asian human canines and premolars. Anthropol Sci 118:191- 198. 2010. doi:10.1537/ase.091006

Gaboutchian et al., 2019. Gaboutchian A.V., Knyaz V.A., Leybova N.A., Petrosyan G.R., Simonyan H.Y. and S.V. Vasilyev. Application Of Photogrammetric Techniques In Palaeoodontological Studies Trough Automated Digital Shape Analysis Of Human Teeth. Int. Arch. Photogramm. Remote Sens. Spatial Inf. Sci., XLII-2/W12, 75-80, https://doi.org/10.5194/isprs-archivesXLII-2-W12-75-2019

Gaboutchian et al., 2020. Gaboutchian, A. V., Knyaz, V. A., Novikov, M. M., Vasilyev, S. V., Leybova, N. A., Korost, D. V., Cherebylo, S. A., and Kudaev, A. A.: Automated Digital Odontometry: Measurement Data Analyses In Cases Of Complicated Dental Morphology, Int. Arch. Photogramm. Remote Sens. Spatial Inf. Sci., XLIII-B2-2020, 851-856, https://doi.org/10.5194/isprs-archives-XLIII-B2-2020851-2020, 2020

Gaboutchian et al, 2021. Gaboutchian, A. V., Knyaz, V. A., Novikov, M. M., Vazyliev, S. V., Korost, D. V., Cherebylo, S. A., and Kudaev, A. A.: COMPARATIVE MORPHOLOGICAL ANALYSIS OF ENAMEL AND DENTIN 
SURFACES' RECONSTRUCTIONS BY MEANS OF AUTOMATED DIGITAL ODONTOMETRY, Int. Arch. Photogramm. Remote Sens. Spatial Inf. Sci., XLIV-2/W1-2021, 67-72, https://doi.org/10.5194/isprs-archives-XLIV-2-W1-202167-2021, 2021.

Hershkovitz et al., 2018. Hershkovitz, I., Weber, G., Quam, R., Duval, M., Grün, R., Kinsley, L., Ayalon, A., Bar-Matthews, M., Valladas, H., Mercier, N., Arsuaga, J., Martinón-Torres, M., Bermúdez de Castro, J-M., Fornai, C., Martín-Francés, L., Sarig, R., May, H., Krenn, V., Slon, V., Mina, W-E. (2018). The earliest modern humans outside Africa. Science. 359. 456-459. 10.1126/science.aap8369

Hillson, 2003. Hillson H. Dental Anthropology. Cambridge University Press, 2003

Kaifu et al, 2015. Kaifu, Y., Kono, R., Sutikna, Th., Saptomo W.E., Jatmiko, Due Awe, R. (2015). Unique Dental Morphology of Homo floresiensis and Its Evolutionary Implications. PloS one. 10. e0141614. 10.1371/journal.pone.0141614

Krenn et al., 2019. Krenn, V., Fornai, C., Wurm, L., Bookstein, F., Haeusler, M., Weber, G. Variation of 3D outer and inner crown morphology in modern human mandibular premolars. American Journal of Physical Anthropology. Vol. 169. 05/2019, doi:10.1002/ajpa.23858

Knyaz, 2012. Knyaz, V. A.: IMAGE-BASED 3D RECONSTRUCTION AND ANALYSIS FOR ORTHODONTIA, Int. Arch. Photogramm. Remote Sens. Spatial Inf. Sci., XXXIX-B3, 585-589, https://doi.org/10.5194/isprsarchives-XXXIX-B3-5852012, 2012.

Knyaz, 2016. Knyaz, V. A. and Gaboutchian, A. V.: PHOTOGRAMMETRY-BASED AUTOMATED MEASUREMENTS FOR TOOTH SHAPE AND OCCLUSION ANALYSIS, Int. Arch. Photogramm. Remote Sens. Spatial Inf. Sci., XLI-B5, 849-855, https://doi.org/10.5194/isprs-archives-XLIB5-849-2016, 2016.

Kono, 2004. Kono R. Molar enamel thickness and distribution patterns in extant great apes and humans: new insights based on a three-dimensional whole crown perspective. Anthropol Sci 112:121-146. 2004. 10.1537/ase.03106

Martin, 1983. Martin L.B. The relationships of the later Miocene Hominoidea. PhD Thesis, University College London, London. 1983.

Naidu and Freer, 2013. Naidu, D., Freer, T. The evidence supporting methods of tooth width measurement: Part I. Vernier calipers to stereophotogrammetry. Australian orthodontic journal. 29. 159-63

Olejniczak et al., 2007. Olejniczak, A., Grine, F., Martin, L. Micro-computed tomography of primate molars: Methodological aspects of three-dimensional data collection. Vertebrate Paleobiology and Paleoanthropology. 01/2007, doi: 10.1007/978-14020-5845-5_7

Olejniczak et al., 2008. Olejniczak, A., Smith, T., Feeney, R., Macchiarelli, R., Mazurier, A., Bondioli, L., Rosas, A., Fortea, J., Rasilla, M., Garcia-Tabernero, A., Radovcić, J., Skinner, M., Toussaint, M., Hublin, J-J. Dental tissue proportions and enamel thickness in Neanderthal and modern human molars. Journal of human evolution. 55. 12-23. 10.1016/j.jhevol.2007.11.004

Ortiz et al., 2019. Ortiz, A., Bailey, Sh., Delgado, M., Zanolli, C., Demeter, F., Bacon, A-M. A distinguishing feature of Pongo upper molars and its implications for the taxonomic identification of isolated hominid teeth from the Pleistocene of Asia. American Journal of Physical Anthropology, Vol. 170, pages 595-612. doi: 10.1002/ajpa.23928

Schwartz and Dean, 2005. Schwartz, G., Dean, M. Sexual dimorphism in modern human permanent teeth. American journal of physical anthropology. 128. 312-7. 10.1002/ajpa.20211

Smith et al., 2018. Smith, T., Houssaye, A., Kullmer, O., Cabec, A., Olejniczak, A., Schrenk, F., Vos, J., Tafforeau, P. Disentangling isolated dental remains of Asian Pleistocene hominins and pongines. PLOS ONE. 13. e0204737. 10.1371/journal.pone. 0204737

Suwa and Kono, 2005. Suwa, G., Kono, R. A micro-CT based study of linear enamel thickness in the mesial cusp section of human molars: Reevaluation of methodology and assessment of within-tooth, serial, and individual variation. Anthropological Science. 113. 273-289. 10.1537/ase.050118.

Suwa et al., 2009. Suwa, G., Kono, R.T., Simpson, S.W., Asfaw, B., Lovejoy, C.O., White T.D. Paleobiological Implications of the Ardipithecus ramidus Dentition. Science, Vol. 326, 69 (2009), doi: $10.1126 /$ science. 1175824

Zilberman et al., 2003. Zilberman O., Huggare J., Parikakis K. Evaluation of the Validity of Tooth Size and Arch Width Measurements Using Conventional and Three-dimensional Virtual Orthodontic Models // Angle Orthod, 2003. No. 73. Pp. 301-306

Zhang et al., 2016. Zhang, F., Suh, K.-J., Lee, K.-M. Validity of Intraoral Scans Compared with Plaster Models: An In-Vivo Comparison of Dental Measurements and 3D Surface Analysis. PLOS ONE. 11. e0157713. 10.1371/journal.pone.0157713

Zubov, 1968. Zubov A.A., "Odontologiya. Metodika antropologicheskikh issledovaniy”, Moscow, Nauka, 200 p.

Zubov, 2006. Zubov A.A. Methodological Handbook for Anthropological Analysis of Odontological Materials. ETNO-ONLINE, Moscow 